\title{
Optimal electric distribution network configuration using adaptive sunflower optimization
}

\author{
Thuan Thanh Nguyen, Ngoc Thiem Nguyen, Trung Dung Nguyen
}

Faculty of Electrical Engineering Technology, Industrial University of Ho Chi Minh City, Ho Chi Minh City, Vietnam

\begin{tabular}{l}
\hline \hline Article Info \\
\hline Article history: \\
Received May 1, 2020 \\
Revised Mar 25, 2021 \\
Accepted Jun 1, 2021 \\
\hline
\end{tabular}

Keywords:

Adaptive sunflower optimization

Distribution system

Network reconfiguration

Power loss

Sunflower optimization

\begin{abstract}
Network reconfiguration (NR) is a powerful approach for power loss reduction in the distribution system. This paper presents a method of network reconfiguration using adaptive sunflower optimization (ASFO) to minimize power loss of the distribution system. ASFO is developed based on the original sunflower optimization (SFO) that is inspired from moving of sunflower to the sun. In ASFO, the mechanisms including pollination, survival and mortality mechanisms have been adjusted compared to the original SFO to fit with the network reconfiguration problem. The numerical results on the 14-node and 33-node systems have shown that ASFO outperforms to SFO for finding the optimal network configuration with greater success rate and better obtained solution quality. The comparison results with other previous approaches also indicate that ASFO has better performance than other methods in term of optimal network configuration. Thus, ASFO is a powerful method for the NR.
\end{abstract}

This is an open access article under the CC BY-SA license.

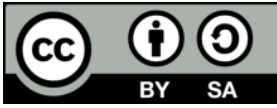

\section{Corresponding Author:}

Thuan Thanh Nguyen

Faculty of Electrical Engineering Technology

Industrial University of Ho Chi Minh City

No. 12 Nguyen Van Bao, Ward 4, Go Vap District, Ho Chi Minh City, Vietnam

Email: nguyenthanhthuan@iuh.edu.vn

\section{INTRODUCTION}

Electric distribution network (EDN) transfers electricity from the transmission system to customers. Because of operating at low voltage level, the EDN's power loss often takes high part with about $70 \%$ in the total losses of distribution and transmission networks [1]. Thus, power loss reduction of distribution network is one of important missions in operating distribution network. There are a lot of techniques for power loss reduction such as capacitor placement, distributed generation installation, increasing cross-section of conductor and operating at high voltage level and network reconfiguration. Whereas, NR is one of the most powerful approaches for decreasing power loss of distribution network. The network reconfiguration approach is achieved by opening and closing switches located in the system. By changing network configuration, load from heavy branches will be transferred to other branches, as a result, and total losses of the system are reduced.

The network reconfiguration has been first solved Merlin and Back in [2] by a branch-and-bound approach. Then several approaches have been demonstrated for the NR problem. Civanlar et al. in [3], a heuristic technique has been used to find the optimal network configuration. The idea of this approach is that an open switch is replaced by other one to decrease power loss. Shirmohammadi and Hong in [4], another heuristic technique has proposed for the network reconfiguration problem, wherein, the branch-and-bound method Merlin and Back in [2] has been improved to determine optimal configuration. Later on, there are a lot of techniques that are inspired from ideals of nature or society phenomena have been proposed for the NR 
problem. The common feature of these methods is that they yield more positive results than heuristic methods. Typical of the above methods must be mentioned to genetic algorithm (GA) [5], [6], particle swarm optimization (PSO) [7]-[9], grey wolf optimization [10], [11], backtracking search algorithm [12], tabu search algorithm (TS) [13], runner root (RRA) [14], symbiotic organisms search (SOS) [15], adaptive shuffled frogs leaping algorithm (ASFLA) [16], improved shuffled frogs leaping algorithm (ISFLA) [17], improved elitist-jaya algorithm (IEJAYA) [18], improved cuckoo search algorithm (ICSA) [19], binary particle swarm gravity search algorithm (BPSO-GSA) [20], and biogeography based optimization (BBO) [21].

Sunflower optimization (SFO) is a new metaheuristic algorithm is first proposed by Gomes et al. [22]. Wherein, SFO is inspired from an idea of moving of sunflower plant to the sun. To solve the optimization problem, a sunflower plant is a candidate solution for the optimization problem and a radiation intensity that the sunflower plant received from the sun is considered as the quality of the candidate solution. Furthermore, a best sunflower plant is considered as the sun and other ones will move to the sun. Gomes et al. in [22], SFO is applied for the problem of damage detection for the composite plate and its performance has been shown to be better than GA. However, the effectiveness of SFO for another problem is still needed to evaluate.

This paper presents a method for optimal network reconfiguration (NR) to minimize power loss using adaptive sunflower optimization (ASFO). In which, ASFO is adjusted from the SFO for adapting to the NR problem. To generate better solution for the NR problem, the all of mechanisms of creating of new sunflower plants such as pollination, survival and mortality mechanisms have been modified. The proposed ASFO has been applied to determine the optimal NR for the 14-node and 33-node distribution systems. The numerical result compared to SFO have shown the outstanding efficiency of the proposed ASFO. Based on the contents of the paper, the highlights of this work can be emphasized is being as:

- SFO is adapted to ASFO for solving the network reconfiguration problem.

- All of mechanisms of creating of new sunflower plants consisting of pollination, survival, and mortality mechanisms have been modified to generate better candidate solutions for the NR problem.

- The performance of ASFO is validated on the 14-node and 33-node systems.

- ASFO is outstanding to SFO for searching optimal NR.

The rest of paper is organized is being as. The problem of network reconfiguration is shown in the below section. The network reconfiguration using adaptive sunflower optimization is shown in section 3 . Section 4 shows the results and discussion. Section 5 presents the main conclusion.

\section{PROBLEM OF NETWORK RECONFIGURATION}

There are many benefits of network reconfiguration such as reduction of power loss, over load and improvement of voltage, and load balance. Wherein, due to high power loss character of distribution level, power loss reduction is considered as one of important goals of network reconfiguration. It is calculated is being as:

$$
P_{\text {loss }}=\sum_{i=1}^{n_{b r}} p_{\text {loss }, i}
$$

Where $P_{\text {loss }}$ and $p_{\text {loss }, i}$ are the power loss of the system and the branch $i$, respectively. $n_{b r}$ is number of branches. Changing the network configuration of distribution system should ensure the following constraints: The radial network configuration: In order to maintain the constraint, (2) should be ensured [13]:

$$
|\operatorname{det}(C)|=1
$$

Where, $\operatorname{det}(C)$ is the $C$ matrix's determinant. $C$ is a connected matrix among branches and nodes of the distribution system. In addition, the obtained network configuration by reconfiguration should not negatively affect to voltage and current profile:

$$
\left\{\begin{array}{l}
V_{j} \geq V_{\text {lo,limit }} ; j=1, \ldots, n_{n o} \\
I_{i} \leq I_{i, \text { ax }} ; i=1, \ldots, n_{b r}
\end{array}\right.
$$

Where, $V_{j}$ is the voltage amplitude of the node $j . V_{l o, l i m i t}$ is allowed minimum voltage amplitude which is often set to 0.95 in per unit. $n_{n o}$ is number of nodes. $I_{i}$ and $I_{i, h i \text {,limit }}$ are the current of the branch $i$ and its rated current. 


\section{NETWORK RECONFIGURATION USING ADAPTIVE SUNFLOWER OPTIMIZATION}

In this section, a method of network reconfiguration using ASFO is presented. Wherein, the original SFO is adjusted to ASFO for generating better solution for the network reconfiguration problem. Details of ASFO for searching the optimal NR are described is being as:

Step 1: generate randomly the population of sunflower plants

$$
S F_{i}=u p+\operatorname{rand}(1, d) .(u p-l o) ; i=1 \div n
$$

Where $S F_{i}$ is the sunflower plant $i . d$ is dimension of the network reconfiguration problem. up and lo are the upper and lower boundaries of the control variables. $n$ is number of sunflowers in the population. The control variables of the network reconfiguration problem present for open switches of the distribution system. Thus, their values are rounded to integer. Then, their adaptive function $\left(A F_{i}\right)$ value consisting of the objective function value and the penalty value of violating constraints is calculated is being as:

$$
A F_{i}=P_{\text {loss }}+K_{P} \cdot\left[\max \left(V_{\text {lo,limit }}-V_{\text {min }}, 0\right)+\max \left(K I_{\text {max }}-K I_{\text {hi,limit }}, 0\right)\right]
$$

Where, $K_{P}$ is penalty factor that is set to 1000 in this work. $V_{\min }$ is minimum voltage of the obtained network configuration. $K I_{\max }$ is maximum load carrying factor of the obtained network configuration. $K I_{\text {hi,limit }}$ is the permitted load carrying factor that is set to 1 .

Step 2: generate new sunflower plants using the pollination mechanism

In the original SFO, the new solutions are generated by using the pollination mechanism is being as:

$$
S F_{i, n e w}=\operatorname{rand}(0,1) \cdot\left(S F_{i}-S F_{i+1}\right)+S F_{i+1} ; i=1 \div R_{p} \cdot n
$$

Where $R_{p}$ is the pollination rate which is set to 0.6 [22]. It can be seen that all sunflowers in the population will tend to move to the sun. The component of difference of the two solutions in the above equation will not produce significant increments to create an entirely new solutions for exploring the search space. Furthermore, to increase the diversity of the control variables, a vector of random numbers are used instead of the random number only. Therefore, in order to create a new solutions for the network reconfiguration problem, the above equation is adjusted is being as:

$$
S F_{i, n e w}=\operatorname{rand}(1, d) \cdot \beta \cdot\left(S F_{i}-S F_{i+1}\right)+S F_{i+1} ; i=1 \div R_{p} \cdot n
$$

Where, $\beta$ is a gain coefficient. Its value depends on the space search of variables. Depending on the scale of the distribution system, the space search of each variable can range from some switches to several dozen switches. So, in this work it is chosen to 4 .

Step 3: generate new sunflower plants using the survival mechanism

In this mechanism of SFO, new sunflower plants are created based on distance between itself to the best sunflower plant $\left(S F_{\text {best }}\right)$ is being as:

$$
S F_{i, n e w}=S F_{i}+\operatorname{rand}(0,1) \cdot\left(\left(S F_{\text {best }}-S F_{i}\right) /\left(\left\|S F_{\text {best }}-S F_{i}\right\|\right)\right) ; i=R_{p} \cdot n \div n \cdot\left(1-R_{d}\right)
$$

Where, $S F_{\text {best }}$ is the best sunflower plant. $\left\|S F_{\text {best }}-S F_{i}\right\|$ is the Euclidean length between plant $i$ and the best plant. $R_{d}$ is a death rate which is set to 0.1 [22]. Similarly to the pollination mechanism, the survival mechanism is adjusted is being as:

$$
S F_{i, \text { new }}=S F_{i}+\operatorname{rand}(1, d) \cdot \beta \cdot\left(\left(S F_{\text {best }}-S F_{i}\right) /\left(\left\|S F_{\text {best }}-S F_{i}\right\|\right)\right) ; i=R_{p} \cdot n \div n \cdot\left(1-R_{d}\right)
$$

Step 4: generate new sunflower plants using the mortality mechanism

The rest sunflower plants are renewed by using random initialization. In ASFO the vector of random numbers are used instead of the random number only in the original SFO is being as:

$$
S F_{i, n e w}=u p+\operatorname{rand}(1, d) \cdot(u p-l o) ; n \cdot\left(1-r_{d}\right) \div n
$$

Step 5: Selection new population of sunflower plants for next generation

All of new sunflower plants are evaluated the adaptive function by using in (5) to obtain the adaptive function value $\left(A F_{i, n e w}\right)$. Then, if new plants have the better quality than the corresponding ones, they will substitute for current sunflower plants is being as: 


$$
\begin{gathered}
S F_{i}=\left\{\begin{array}{c}
S F_{i, n e w} ; \text { if } A F_{i, n e w}<A F_{i} \\
S F_{i} ; \text { otherwise }
\end{array}\right. \\
A F_{i}=\left\{\begin{array}{c}
A F_{i, n e w} ; \text { if } A F_{i, n e w}<A F_{i} \\
A F_{i} ; \text { otherwise }
\end{array}\right.
\end{gathered}
$$

Step 6: stop searching the optimal solution

The searching process from step 2 to step 5 will be performance until the maximum number of generations $\left(M G_{\max }\right)$ reaches. The flowchart of ASFO for finding the optimal network configuration is shown in Figure 1.

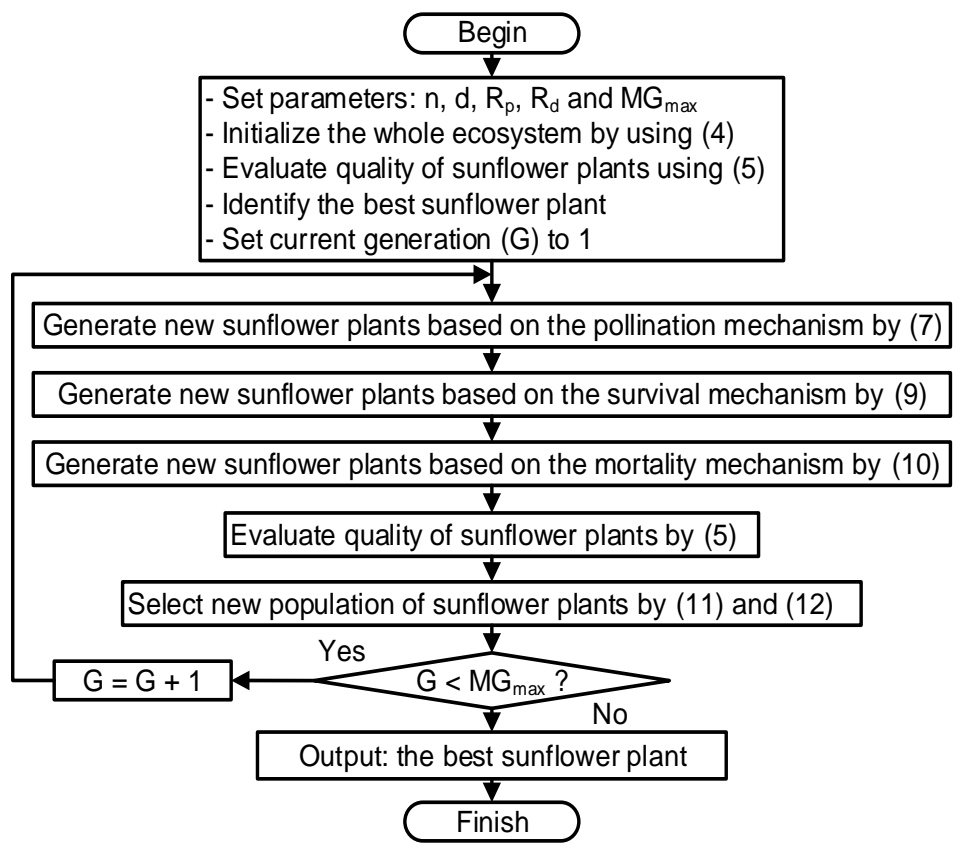

Figure 1. Flowchart of ASFO for network reconfiguration

\section{RESULTS AND DISCUSSION}

To demonstrate effectiveness of ASFO, two distribution systems consisting of 14-node and 33-node networks are used to find the optimal NR. The performance of ASFO is compared to the SFO in criteria such as maximum $\left(A F_{\text {max }}\right)$, minimum $\left(A F_{\text {min }}\right)$, mean $\left(A F_{\text {mean }}\right)$ and standard deviation $(S T D)$ values of the adaptive function gained in 50 runs as well as the mean run times $\left(T_{\text {run }}\right)$ [23]. Both of these methods have coded in Matlab 2016a and run on the same personal computer of $4 \mathrm{G}$ random access memory (RAM) and intel core i5, $2.4 \mathrm{Gh}$. In addition, the obtained results from ASFO are also compared with other methods in literature to show the reliability of the proposed method. The parameters of ASFO and SFO consisting of $n$ and $M G_{\max }$ are chosen to $\{10,100\}$ for the 14 -node system and $\{20,150\}$ for the 33 -node system.

\subsection{The 14-node network}

The system consists of three open switches as shown in Figure 2 [5]. The initial power loss of the system is $511.4356 \mathrm{~kW}$. The optimal network configuration obtained by the proposed ASFO method are shown in Table 1. The switches (SW) consisting of $\{6-12-14\}$ are opened substituting for $\{14-15-16\}$ in the optimal network configuration. This changing has caused power loss $\left(P_{\text {loss }}\right)$ of $466.1267 \mathrm{~kW}$ and minimum voltage $\left(V_{\min }\right)$ of 0.9716 p.u. Both of these indicators are better than those of the initial network configuration. Wherein, the former is $45.3089 \mathrm{~kW}$ lower and the latter is 0.0023 higher than those of the initial network configuration. Furthermore, the voltage amplitude of nodes shown in Figure 3 sends a message that voltage improvement gained by network reconfiguration is remarkable with most of node voltages have been increased. The optimal network configuration gained by ASFO is identical to that of GA [5], BPSO-GSA [20] and TS [13]. These comparisons demonstrate the reliability of the ASFO for the network reconfiguration problem. 


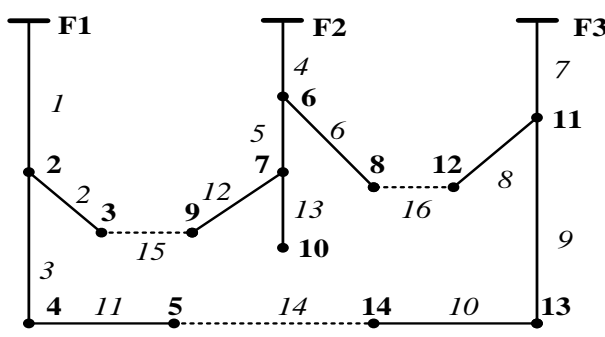

Figure 2. The first test 14-node system

Table 1. The obtained results of ASFO, SFO, and previous methods for the 14-node network

\begin{tabular}{ccccccc}
\hline Item & None & ASFO & SFO & GA [5] & BPSO-GSA [20] & TS [13] \\
\hline$S W$ & $14-15-16$ & $6-12-14$ & $6-12-14$ & $6-12-14$ & $6-12-14$ & $6-12-14$ \\
$P_{\text {loss }}(\mathrm{kW})$ & 511.4356 & 466.1267 & 466.1267 & 466.1267 & 466.1267 & 466.1267 \\
$V_{\text {min }}($ p.u. $)$ & 0.9693 & 0.9716 & 0.9716 & 0.9716 & 0.9716 & 0.9716 \\
$A F_{\text {max }}$ & - & 466.1267 & 511.44 & - & - & - \\
$A F_{\min }$ & - & 466.1267 & 466.1267 & - & - & - \\
$A F_{\text {mean }}$ & - & 466.1267 & 475.7757 & - & - & - \\
$S T D$ & - & 0 & 10.7152 & - & - & - \\
$T_{\text {run }}(\mathrm{s})$ & - & 2.9475 & 2.6288 & - & - & - \\
\hline
\end{tabular}

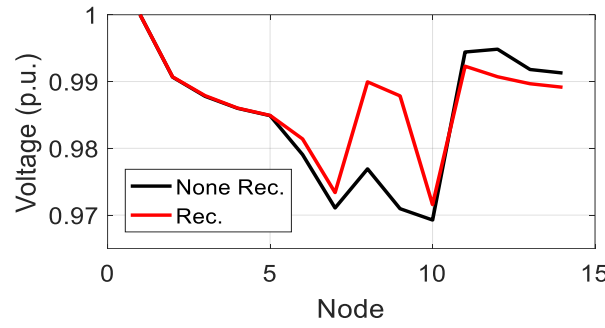

Figure 3. The voltages of the initial and optimal configurations of the 14-node network

In comparison with $\mathrm{SFO}$, the indicators such as $A F_{\text {max }}, A F_{\text {min }}, A F_{\text {mean }}$ and $S T D$ values of the adaptive function gained in 50 runs show that ASFO outperforms to SFO. Although both of ASFO and SFO have searched out the optimal network configuration (shown by the same $A F_{\min }$ value), the $A F_{\text {max }}$, and $A F_{\text {mean }}$ values of ASFO are much lower compared to SFO. In which, the $A F_{\text {max }}$ and $A F_{\text {mean }}$ values of ASFO are 45.3133 and 9.649 lower compared to those of SFO. In addition, STD value of ASFO is much lower compared to that of SFO. Figure 4 (a) shows that ASFO has achieved the optimal NR in all of 50 runs with STD of 0 while SFO has found the optimal solution in 21 per 50 runs with STD of 10.7152 . The maximum, mean and minimum convergence characters of both methods are shown in Figure 4 (b). Figure shows that ASFO converges to lower value and lower convergence generations compared to SFO. The run times of ASFO is 0.3187 seconds (s) higher than that of SFO. These results show that the improvement of ASFO is remarkable to the NR problem.

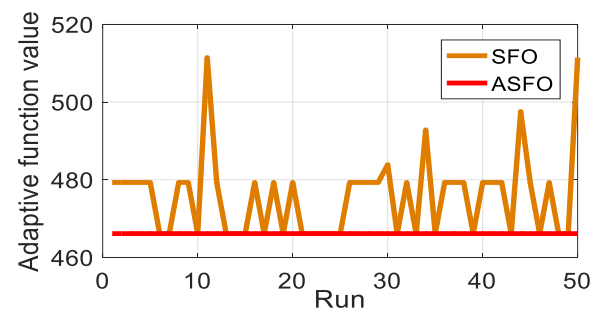

(a)

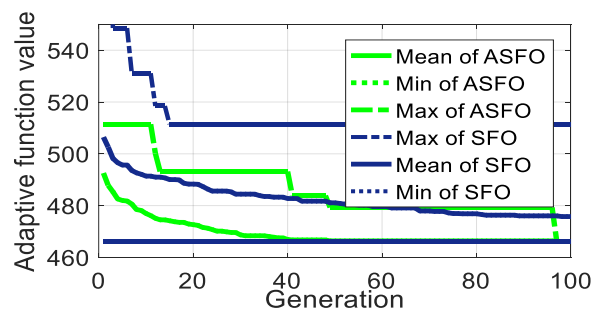

(b)

Figure 4. The performance of ASFO and SFO for the 14-node network, (a) obtained adaptive function value, (b) convergence curves 


\subsection{The 33-node network}

The EDN in Figure 5 consists of five open switches of $\{33-34-35-36-37\}$ [24]. The maximum branch current limit is set to $255 \mathrm{~A}$ [25], [26]. The power loss, maximum load carrying factor and minimum voltage of the system in case of none reconfiguration are $202.6863 \mathrm{~kW}, 0.8250$ and 0.9131 p.u.

The optimal network configuration achieved by the ASFO method are shown in Table 2. The switches (SW) consisting of $\{7-9-14-28-32\}$ are opened substituting for $\{33-34-35-36-37\}$ in the optimal network configuration. This changing has caused $P_{\text {loss }}$ of $139.9823 \mathrm{~kW}$ and $V_{\min }$ of 0.9412 . Both of these indicators are better than those of the initial network configuration. Wherein, the former is $62.704 \mathrm{~kW}$ lower and the latter is 0.0281 greater than those of the initial network configuration. In addition, the voltage amplitude of nodes and current of branches shown in Figure 6 show that voltage and current improvements gained by network reconfiguration are remarkable with increasing of most of node voltages and decreasing of most of branch currents.

The optimal network configuration gained by ASFO is identical to that of ASFLA [16], SOS [15] and IEJAYA [18]. The result of ASFO is better than that of BPSO-GSA [20] and GA [27]. Wherein, $P_{\text {loss }}$ value obtained by ASFO is 1.2248 and 23.1648 lower than that of BPSO-GSA [20] and GA [27]. The $V_{\min }$ value of ASFO is also 0.0034 and 0.0333 higher than that of BPSO-GSA [20] and GA [27]. Compared with ISFLA [17], PSO [7], and BBO [21], the $P_{\text {loss }}$ value obtained by ASFO is $0.428,0.0223$ and 0.428 higher than that of above methods but the $V_{\min }$ value of ASFO is $0.0034,0.0117$ and 0.0034 higher than that of the ISFLA [17], PSO [7], and BBO [21] methods. These comparisons have demonstrated once again that the reliability of the ASFO for the network reconfiguration problem.

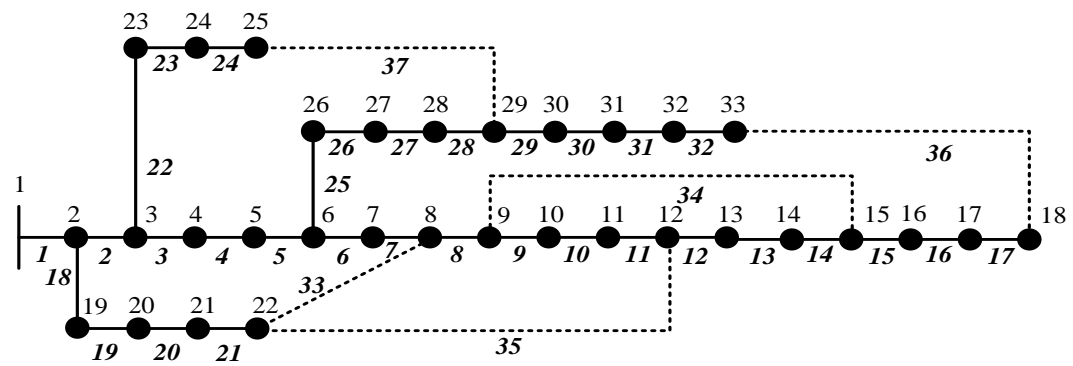

Figure 5. The second test 33-node network

Table 2. The obtained results of ASFO, SFO, and other methods for the 33-node network

\begin{tabular}{cccccccccc}
\hline Method & $S W$ & $P_{\text {loss }}(\mathrm{kW})$ & $V_{\min }(\mathrm{p})$. & $K I_{\max }$ & $A F_{\max }$ & $A F_{\min }$ & $A F_{\operatorname{mean}}$ & $S T D$ & $T_{\text {run }}(\mathrm{s})$ \\
\hline None rec. & $33-34-35-36-37$ & 202.6863 & 0.9131 & 0.8250 & - & - & - & - \\
ASFO & $7-9-14-28-32$ & 139.9823 & 0.9412 & 0.8126 & 164.309 & 148.7392 & 153.6637 & 4.0604 & 7.6666 \\
SFO & $7-10-14-27-32$ & 144.0295 & 0.9398 & 0.8135 & 197.927 & 154.2448 & 174.6159 & 9.3234 & 5.7231 \\
ASFLA [16] & $7-9-14-28-32$ & 139.9823 & 0.9412 & 0.8126 & - & - & - & - & - \\
BPSO-GSA [20] & $7-11-14-32-37$ & 141.2071 & 0.9378 & - & - & - & - & - & - \\
SOS [15] & $7-9-14-28-32$ & 139.9823 & 0.9412 & - & - & - & - & - \\
ISFLA [17] & $7-9-14-32-37$ & 139.5543 & 0.9378 & - & - & - & - & - & - \\
IEJAYA [18] & $7-9-14-28-32$ & 139.9823 & 0.9412 & - & - & - & - & - & - \\
PSO [7] & $7-14-32-35-37$ & 139.9600 & 0.92946 & - & - & - & - & - & - \\
BBO [21] & $7-9-14-32-37$ & 139.5543 & 0.9378 & - & - & - & - & - & - \\
GA [27] & $7-12-31-35-37$ & 163.1471 & 0.9079 & - & - & - & - & - & - \\
\hline
\end{tabular}

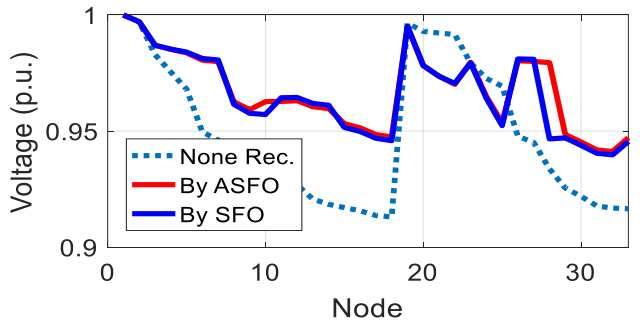

(a)

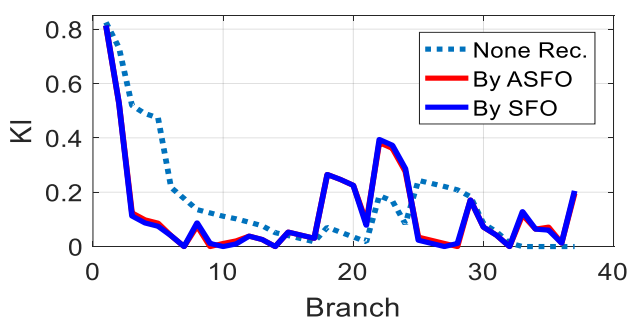

(b)

Figure 6. The voltages and currents achieved by ASFO and SFO for the 33-node network, (a) voltage profile, (b) current profile 
In comparison with SFO, in 50 runs, SFO has only searched the network configuration with power loss of $144.0295 \mathrm{~kW}$ that is $4.0472 \mathrm{kw}$ higher than that of ASFO and the $V_{\min }$ value is 0.0014 higher than that of ASFO. In addition, the indicators consisting of $A F_{\text {max }}, A F_{\text {min }}, A F_{\text {mean }}$ and $S T D$ values of ASFO are much lower compared to SFO. In which, these values of ASFO are 33.618, 5.5056, 20.9522 and 5.263 lower compared to those of SFO. Figure 7 (a) shows that ASFO has gained lower adaptive function value than that of SFO in most of runs. The maximum, mean and minimum convergence characters of both methods are shown in Figure 7 (b). Figure 7 shows that ASFO converges to lower value and lower convergence generations compared to SFO. The $T_{\text {run }}$ value of ASFO is $1.9435 \mathrm{~s}$ higher than that of SFO. These achieved results presents that ASFO is better than SFO for the NR problem.

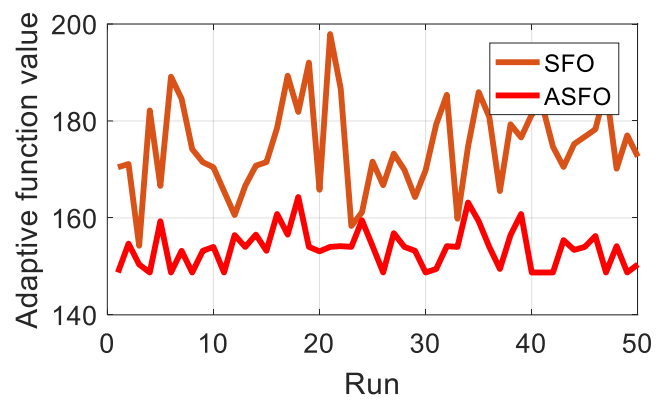

(a)

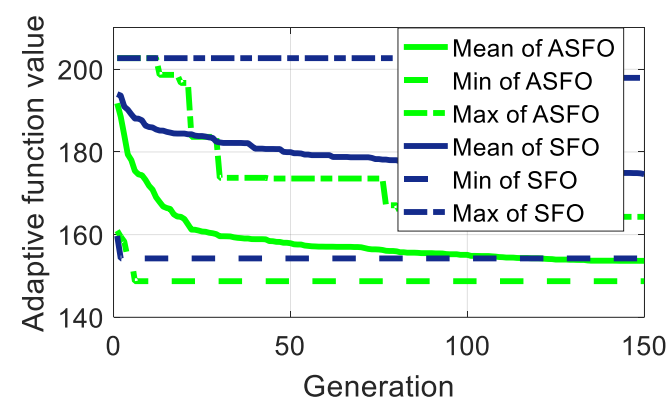

(b)

Figure 7. The performance of ASFO and SFO for the 33-node network, (a) obtained adaptive function value, (b) convergence curves

\section{CONCLUSION}

In this work, the network reconfiguration problem for power loss reduction has been successfully solved by using the proposed ASFO method. Wherein, to increase the efficiency of ASFO for the NR problem, the ASFO search mechanisms including pollination, survival, and mortality mechanisms have been adjusted compared to the original SFO. In which, the pollination and survival mechanisms has been added gain factors and all of three mechanisms vector of random numbers have been used to replace for a random number. The performance of ASFO has been validated on the 14-node and 33-node systems. The obtained results compared to SFO show that ASFO has better performance than SFO in terms of the optimal network configuration and maximum, minimum, mean and STD values of the adaptive function in several runs. The compared results to other techniques have also shown that ASFO is in one of the effective approach for the network reconfiguration problem. Future work may consider the performance of AFO for the NR for other purposes or other optimization problems in power systems.

\section{REFERENCES}

[1] O. Badran, S. Mekhilef, H. Mokhlis, and W. Dahalan, "Optimal reconfiguration of distribution system connected with distributed generations: A review of different methodologies," Renewable and Sustainable Energy Reviews, vol. 73, pp. 854-867, August 2015, doi: 10.1016/j.rser.2017.02.010.

[2] A. Merlin and H. Back, "Search for a minimal loss operating spanning tree configuration in an urban power distribution system," Proceeding in 5th power system computation conf (PSCC), Cambridge, UK, vol. 1, pp. 1-18, 1975.

[3] S. Civanlar, J. J. Grainger, H. Yin, and S. S. H. Lee, "Distribution feeder reconfiguration for loss reduction," in IEEE Transactions on Power Delivery, vol. 3, no. 3, pp. 1217-1223, July 1988, doi: 10.1109/61.193906.

[4] D. Shirmohammadi and H. W. Hong, "Reconfiguration of electric distribution networks for resistive line losses reduction," in IEEE Transactions on Power Delivery, vol. 4, no. 2, pp. 1492-1498, April 1989, doi: $10.1109 / 61.25637$.

[5] J. Z. Zhu, "Optimal reconfiguration of electrical distribution network using the refined genetic algorithm," Electric Power Systems Research, vol. 62, no. 1, pp. 37-42, 2002, doi: 10.1016/S0378-7796(02)00041-X.

[6] P. Subburaj, K. Ramar, L. Ganesan, and P. Venkatesh, "Distribution System Reconfiguration for Loss Reduction using Genetic Algorithm," Journal of Electrical Systems, vol. 2, no. 4, pp. 198-207, 2006.

[7] D. Kumar, A. Singh, S. K. Mishra, R. C. Jha, and S. R. Samantaray, "A coordinated planning framework of electric power distribution system: Intelligent reconfiguration," International Transactions on Electrical Energy Systems, vol. 28, no. 6, pp. 1-20, 2018, doi: 10.1002/etep.2543.

[8] K. K. Kumar, N. Venkata, and S. Kamakshaiah, "FDR particle swarm algorithm for network reconfiguration of distribution systems," Journal of Theoretical and Applied Information Technology, vol. 36, no. 2, pp. 174-181, 2012. 
[9] A. A. Firdaus, O. Penangsang, A. Soeprijanto, and U. P. Dimas Fajar, "Distribution network reconfiguration using binary particle swarm optimization to minimize losses and decrease voltage stability index," Bulletin of Electrical Engineering and Informatics, vol. 7, no. 4, pp. 514-521, 2018, doi: 10.11591/eei.v7i4.821.

[10] A. V. S. Reddy, M. D. Reddy, and M. S. K. Reddy, "Network reconfiguration of distribution system for loss reduction using GWO algorithm," International Journal of Electrical and Computer Engineering, vol. 7, no. 6, pp. 3226-3234, 2017, doi: 10.11591/ijece.v7i6.pp3226-3234.

[11] H. Hamour, S. Kamel, L. Nasrat, and J. Yu, "Distribution Network Reconfiguration Using Augmented Grey Wolf Optimization Algorithm for Power Loss Minimization," 2019 International Conference on Innovative Trends in Computer Engineering (ITCE), 2019, pp. 450-454, doi: 10.1109/ITCE.2019.8646595.

[12] T. T. Nguyen, "Optimal distribution network configuration using improved backtracking search algorithm," Telkomnika (Telecommunication Computing Electronics and Control), vol. 19, no. 1, pp. 301-309, 2021, doi: 10.12928/TELKOMNIKA.v19i1.16773.

[13] A. Y. Abdelaziz, F. M. Mohamed, S. F. Mekhamer, and M. A. L. Badr, "Distribution system reconfiguration using a modified Tabu Search algorithm," Electric Power Systems Research, vol. 80, no. 8, pp. 943-953, 2010, doi: 10.1016/j.epsr.2010.01.001.

[14] T. T. Nguyen, "Electric distribution network reconfiguration for power loss reduction based on runner root algorithm," International Journal of Electrical and Computer Engineering (IJECE), vol. 10, no. 5, pp. 5016-5024, 2020, doi: 10.11591/ijece.v10i5.pp5016-5024.

[15] T. L. Duong and T. T. Nguyen, "Network Reconfiguration for an Electric Distribution System with Distributed Generators based on Symbiotic Organisms Search," Engineering, Technology \& Applied Science Research, vol. 9, no. 6, pp. 4925-4932, 2019.

[16] A. Onlam, D. Yodphet, R. Chatthaworn, C. Surawanitkun, A. Siritaratiwat, and P. Khunkitti, "Power Loss Minimization and Voltage Stability Improvement in Electrical Distribution System via Network Reconfiguration and Distributed Generation Placement Using Novel Adaptive Shuffled Frogs Leaping Algorithm," Energies, vol. 12, no. 3, p. 553, 2019, doi: 10.3390/en12030553.

[17] J. Siahbalaee, N. Rezanejad, and G. B. Gharehpetian, "Reconfiguration and DG Sizing and Placement Using Improved Shuffled Frog Leaping Algorithm," Electric Power Components and Systems, vol. 47, no. 16-17, pp. 1475-1488, 2019, doi: 10.1080/15325008.2019.1689449.

[18] U. Raut and S. Mishra, "An improved Elitist-Jaya algorithm for simultaneous network reconfiguration and DG allocation in power distribution systems," Renewable Energy Focus, vol. 30, pp. 92-106, 2019, doi: 10.1016/j.ref.2019.04.001.

[19] T. T. Nguyen, "Optimization of distribution network configuration with multi objective function based on improved cuckoo search algorithm," Bulletin of Electrical Engineering and Informatics, vol. 9, no. 4, pp. 16851693, 2020, doi: 10.11591/eei.v9i4.1886.

[20] A. Fathy, M. El-Arini, and O. El-Baksawy, "An efficient methodology for optimal reconfiguration of electric distribution network considering reliability indices via binary particle swarm gravity search algorithm," Neural Computing and Applications, vol. 30, pp. 2843-2858, 2018, doi: 10.1007/s00521-017-2877-z.

[21] M. Mosbah, R. Zine, S. Arif, and R. d. Mohammedi, "Optimum Distribution Network Reconfiguration in Presence DG Unit Using BBO Algorithm,” Journal Electrical Systems, vol. 14, no. 4, pp. 180-189, 2018.

[22] G. F. Gomes, S. S. da Cunha, and A. C. Ancelotti, "A sunflower optimization (SFO) algorithm applied to damage identification on laminated composite plates," Engineering with Computers, vol. 35, no. 2, pp. 619-626, 2019, doi: 10.1007/s00366-018-0620-8

[23] T. T. Nguyen, D. N. Vo, and B. H. Dinh, "An effectively adaptive selective cuckoo search algorithm for solving three complicated short-term hydrothermal scheduling problems," Energy, vol. 155, pp. 930-956, July 2018, doi: 10.1016/j.energy.2018.05.037.

[24] M. E. Baran and F. F. Wu, "Network reconfiguration in distribution systems for loss reduction and load balancing," in IEEE Transactions on Power Delivery, vol. 4, no. 2, pp. 1401-1407, April 1989, doi: 10.1109/61.25627.

[25] S. Ghasemi and J. Moshtagh, "Radial distribution systems reconfiguration considering power losses cost and damage cost due to power supply interruption of consumers," International Journal on Electrical Engineering and Informatics, vol. 5, no. 3, pp. 297-315, 2013.

[26] J. Moshtagh and S. Ghasemi, "Optimal Distribution System Reconfiguration Using Non- dominated Sorting Genetic Algorithm ( NSGA-II )," Journal of Operation and Automation in Power Engineering, vol. 1, no. 1, pp. 297-315, 2013.

[27] M. Abdelaziz, "Distribution network reconfiguration using a genetic algorithm with varying population size," Electric Power Systems Research, vol. 142, pp. 9-11, January 2017, doi: 10.1016/j.epsr.2016.08.026. 